\title{
La opinión de los graduados europeos sobre la universidad cinco años después de haber finalizado sus estudios
}

\author{
José-Miguel Carot \\ Andrea Conchado
}

Universidad Politécnica de Valencia. CEGES-LMPF*

jcarot@upv.es; anconpei@upvnet.upv.es

José-Ginés Mora

University of London. Institute of Education

josegines@upv.es

Luis E. Vila

Universidad de Valencia

luis.vila@uv.es

\section{Resumen}

En el contexto actual, en el que la sociedad del conocimiento reclama nuevas capacidades de los graduados universitarios para adaptarse a las demandas del mercado laboral, ha surgido la figura del "profesional flexible». Las universidades no son ajenas a los cambios que, en este sentido, se están produciendo, y se están planteando modificaciones y rediseños de los planes de estudio. En este trabajo, se pretende describir la visión que los graduados universitarios tienen de la universidad transcurridos cinco años desde que finalizaron sus estudios. La información que, desde su experiencia educativa, tienen los titulados es fundamental para los gestores educativos en la tarea de diseño de planes de estudio o políticas educativas. Los datos utilizados proceden de la encuesta REFLEX.

Palabras clave: competencias; Espacio Europeo de Educación Superior; estudios universitarios; titulado superior.

Abstract. The Opinion of European Graduates About the University Five Years After Completing Their Education

In today's knowledge society, in which university graduates are required to obtain new abilities to adjust to the demands of the labour market, the figure of the "flexible professional" has emerged. Universities are not immune to these changes and are proposing

* CEGES-LMPF es un grupo de investigadores basado en el CEGES de la Universidad Politécnica de Valencia (UPV) (www.ceges.upv.es). Está coordinado por José-Ginés Mora y formado por Lourdes Badillo (Universidad Politécnica de Cartagena), José-Miguel Carot (UPV), Andrea Conchado (UPV), Jose-María Nyssen (ANECA) y Luis E. Vila (Universidad de Valencia). 
modifications to the curricula. In this paper, we attempt to depict the views of university graduates regarding their university five years after completing their studies. Information about graduates' educational experience is crucial to educational managers in charge of designing curricula and educational policies. The data used in this paper are obtained from the REFLEX Survey.

Key words: Competencies; European Higher Education Area; University Studies; Higher Education Graduate.

\section{Sumario}

1. Introducción 5. Comportamiento de los estudiantes

\section{El proyecto REFLEX}

3. Evaluación de los graduados sobre los métodos de enseñanza-aprendizaje de su universidad

4. Características de la carrera durante la carrera

6. Evaluación de los estudios realizados

7. Las competencias en España

8. Conclusiones

Referencias bibliográficas

\section{Introducción}

El mercado de trabajo europeo actual se enmarca en la llamada «sociedad del conocimiento", la cual no sólo exige de los graduados universitarios conocimientos y destrezas a nivel profesional, sino también la capacidad de adaptarse a nuevos ámbitos de desarrollo profesional no necesariamente relacionados con su campo específico de estudio (Schomburg, 2007; Mora y CEGES-LMPF, 2008). De esta forma, surge la figura de un nuevo tipo de trabajador: el «profesional flexible». Como consecuencia de ello, los países europeos han experimentado la necesidad de introducir cambios en sus sistemas educativos (Knabb y Stoddard, 2005; Kellerman, 2007).

En un momento como el actual, en que las universidades se plantean el diseño de nuevos planes de estudio, este artículo pretende proporcionar información de especial relevancia para los gestores educativos universitarios, considerando como tales a los responsables de la gestión y la organización de los estudios en las universidades (European Commission, 2005). Esto incluye, lógicamente, a todos los profesores universitarios que deben estar interesados en conocer cuál es el destino y la opinión de sus graduados, especialmente en aquellos aspectos que más tienen que ver con la formación que han recibido durante su permanencia en la universidad. Para ello, utilizando datos procedentes del proyecto internacional REFLEX (Research on the Flexible Professional in the Knowledge Society), se examina las respuestas de más de 40.000 jóvenes graduados europeos (5.500 españoles) que, cinco años después de finalizar sus estudios universitarios, evalúan los métodos de enseñanza-aprendizaje utilizados en su universidad, algunas de las características de su carrera, su dedicación a la misma y cómo ésta ha contribuido a su desarrollo vital y profesional (Vila, García-Aracil y Mora, 2007). Adicionalmente, en el caso de España, se examina 
cómo los estudios universitarios han favorecido el desarrollo de las competencias profesionales de los graduados. Pese a la importancia del tema, el resto de países participantes en el proyecto REFLEX no consideró este último aspecto a la hora de entrevistar a sus titulados.

Para los centros de educación superior, es crucial saber cómo ayudar a los graduados universitarios a satisfacer la amplia gama de exigencias requeridas por los puestos de trabajo. Esta cuestión puede resolverse desde diferentes perspectivas y a diferentes niveles. En el pasado, los sistemas de educación superior se han desarrollado en cada país según su propia lógica interna, o por lo menos siguiendo su propia trayectoria. Es importante darse cuenta de hasta qué punto este proceso, así como otros recientes desarrollos en los sistemas de educación superior nacionales, están dando lugar a una visión común de lo exigido a los graduados universitarios en la sociedad del conocimiento, y el punto hasta el que la política de la enseñanza está guiada por otros objetivos, como la autonomía académica o la emancipación y la igualdad de oportunidades. El caso de cada país es diferente y variado. Los distintos sistemas educativos y currículos pueden estar enfocados en proporcionar a los graduados las competencias profesionales exigidas por los distintos puestos de trabajo. Sin embargo, conocer cuáles son dichas competencias es una labor complicada, ya que pueden variar según el campo ocupacional, el campo de estudio e incluso el país. Sólo es posible saber hasta qué punto las competencias proporcionadas por la universidad satisfacen los requerimientos de los empleos relacionando las competencias creadas con la actuación, tanto de los graduados universitarios como de las organizaciones para las que trabajan.

Así pues, en el proceso de convergencia en el que estamos inmersos hacia un espacio europeo de educación superior (EEES), es de gran importancia conocer con la máxima precisión la situación en la que se encuentra la universidad para diseñar los nuevos planes de estudio. La adaptación al EEES, como es bien conocido, implica un diseño de las materias basado en competencias (Hartog, 2001). Esto afecta a los métodos de enseñanza-aprendizaje, a la metodología de evaluación y conlleva un cambio en el comportamiento de los estudiantes durante los estudios (Belfield, Bullock y Fielding, 1999; Meng y Heike, 2005). Tener esta información es esencial para los agentes implicados en el rediseño de los planes de estudio que deben adaptar el sistema actual a las nuevas exigencias del EEES.

\section{El proyecto REFLEX}

El proyecto REFLEX (Reflex, 2009) es una iniciativa que forma parte del $6^{\circ}$ Programa Marco de la Unión Europea, en el cual han colaborado trece países europeos: Italia, España, Francia, Austria, Alemania, Países Bajos, Reino Unido, Finlandia, Noruega, República Checa, Suiza, Bélgica y Estonia. Su ámbito de estudio se enmarca en los graduados cinco años después de finalizar su carrera universitaria en el año académico 1999-2000. La gestión y coordinación del proyecto a nivel europeo se ha llevado a cabo por el Research Centre for Education and the Labour Market, de la Universidad de Maastricht. En España, 
el coordinador del proyecto ha sido el Centro de Estudios en Gestión de la Educación Superior (CEGES), de la Universidad Politécnica de Valencia, que ha contado con la colaboración de la Agencia Nacional de Evaluación de la Calidad y Acreditación (ANECA). Adicionalmente, también se dispone de datos de algunos países latinoamericanos, de los cuales, en este trabajo, se presentan los referidos a México.

El objetivo global del proyecto REFLEX se centra en tres cuestiones generales e interrelacionadas:

1. ¿Qué competencias requieren los graduados en educación superior para integrarse en la sociedad del conocimiento?

2. ¿Qué papel desempeñan las universidades en el desarrollo de dichas competencias?

3. ¿Cuál es el grado de consecución de las expectativas de los graduados con sus trabajos y de qué forma pueden resolverse los desajustes entre sus expectativas y las características de su trabajo?

El instrumento clave del proyecto es una encuesta de amplio cuestionario realizada a los graduados universitarios cinco años después de la graduación. En este tiempo, cabe esperar que los graduados se hayan incorporado ya al mercado laboral y, por tanto, que puedan comparar lo que se les demanda y lo que se les ofrece desde su puesto de trabajo con la formación que recibieron en la universidad.

\section{La encuesta REFLEX}

La encuesta REFLEX es un instrumento de medición que surgió a partir de la experiencia obtenida en el desarrollo de otro proyecto precedente muy semejante metodológicamente, el proyecto CHEERS (Careers of Higher Education Graduates in Europe), financiado igualmente por la UE. En su diseño y mejora han participado quince universidades europeas, entre las que se encuentra la Universidad Politécnica de Valencia. El cuestionario de la encuesta REFLEX se estructura de la forma siguiente:

A. Carrera en la que te graduaste en 1999-2000.

B. Otras experiencias en el ámbito educativo.

C. Transición de los estudios al trabajo.

D. Primer trabajo desde la graduación.

E. Historia y situación actual.

F. Trabajo actual.

G. Organización en la que trabajas.

H. Competencias.

I. Evaluación de la carrera.

J. Valores y orientaciones.

K. Información personal. 
En el cuestionario español, se incluyó una variable en el apartado H («Competencias») que no contenían el resto de cuestionarios europeos. Esta variable mide en qué grado ha contribuido la carrera a formar las competencias del titulado. En el último apartado de este trabajo, se analizará esta información (únicamente para el caso español) como una dimensión adicional que completa la visión con que la universidad es percibida por los egresados cinco años después de finalizados sus estudios. Finalmente, se pretende analizar el «salto» que existe entre el nivel competencial que el estudiante poseía al salir de su universidad y el que posee cinco años después de graduarse, es decir, una vez integrado en el mercado de trabajo. Adicionalmente, este estudio refleja la visión que los graduados proyectaron de su experiencia educativa universitaria a través de las respuestas que ofrecieron a una serie de cuestiones relacionadas con los diversos métodos de enseñanza y aprendizaje utilizados, las características más destacables de la carrera cursada, el comportamiento de los propios graduados cuando eran estudiantes, las horas que dedicaban a estudiar y su propensión a comenzar estudios adicionales a los de la carrera en que se graduaron.

A pesar de que, en el proyecto, han participado trece países europeos y se poseen datos de varios países latinoamericanos, con el objetivo de simplificar la presentación de resultados, se han escogido datos referidos sólo a algunos países. Se han elegido, además de España, tres países representativos de los tres modelos tradicionales de universidades: el humboldtiano (Alemania), el napoleónico (Francia) y el anglosajón (Reino Unido); además, se presentan los datos de México como país no europeo cuyo modelo de universidad se ve influenciado por diversas tendencias, incluida la norteamericana. La encuesta realizada fue contestada por unos 40.000 graduados europeos, 5.500 de los cuales eran españoles. Con el objetivo de sintetizar al máximo la información, se ha reducido el conjunto de titulaciones a ocho grandes áreas de estudio: educación, humanidades y arte, ciencias sociales, economía y empresa, derecho, ingeniería y arquitectura, salud y ciencias experimentales.

\section{Evaluación de los graduados sobre los métodos de enseñanza-aprendizaje de su universidad}

Los graduados evaluaron el énfasis que se hacía durante sus estudios en una serie de métodos de enseñanza, aprendizaje y evaluación mediante una escala Likert de 5 puntos, donde 1 significaba nada y 5, mucho. A través de la figura 1, que presenta información relativa al conjunto de los países examinados en este artículo, se muestra el porcentaje de titulados que respondieron que el método de aprendizaje considerado en cada caso era bastante o muy relevante en sus estudios, es decir, que evaluaron su importancia con un valor 4 o 5 de la escala. Una amplia mayoría de los graduados encuestados señalaba que, en sus carreras respectivas, se hacía bastante o mucho énfasis en la "Asistencia a clase» (68\%). Los dos siguientes aspectos más relevantes fueron «Trabajos escritos» (58\%) y «Teorías, conceptos y paradigmas» (56\%). Por el contrario, resultaron muy bajas las proporciones de graduados que afirmaron que, en sus estudios 
universitarios, se hacía bastante o mucho énfasis en la «Realización de pruebas tipo test» (20\%) y en la «Participación en proyectos de investigación» (21\%), sugiriendo que estos métodos, de forma general, son los menos utilizados en la universidad.

Los datos mostrados en la figura 1 sólo sirven para ofrecer una idea global sobre los aspectos a los que, de modo general, se ha dado más importancia. Sin embargo, un estudio pormenorizado muestra notables discrepancias entre países, puesto que refleja las diferencias intrínsecas entre los distintos modelos de universidad. En este sentido, la tabla 1 muestra una interesante doble comparación entre los países examinados en este estudio.

Figura 1. Porcentaje de graduados que han señalado que, en su carrera, se hacía bastante o mucho énfasis en cada uno de los distintos métodos de enseñanza-aprendizaje
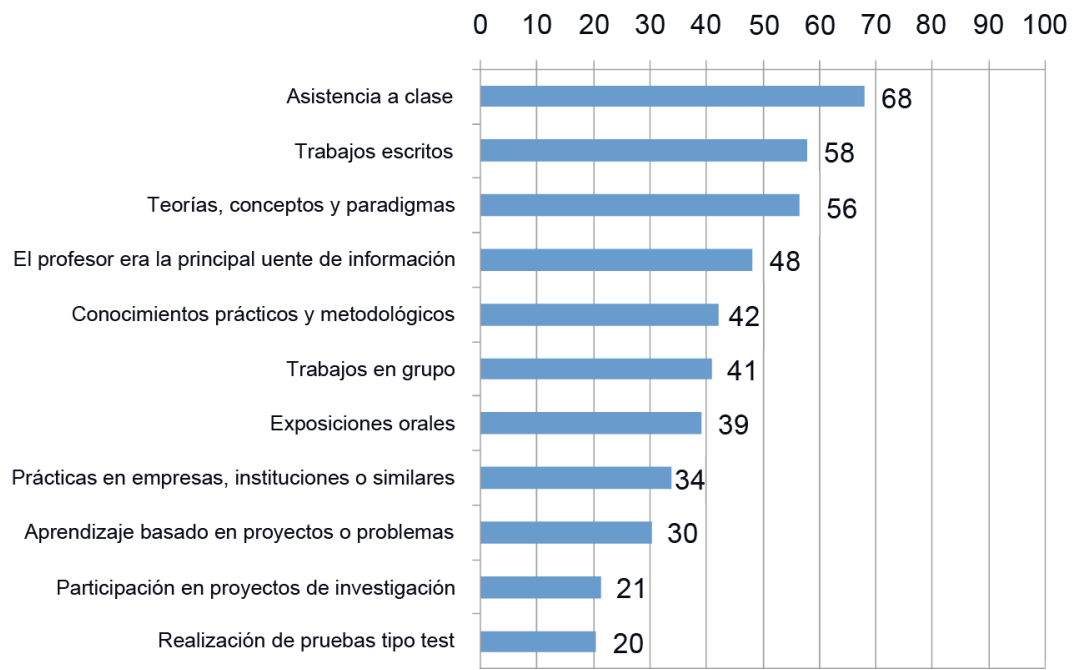

Fuente: elaboración propia.

Tabla 1. Aspectos de los métodos de enseñanza-aprendizaje desagregados por países. Porcentaje de encuestados que han respondido «bastante» $\mathrm{o}$ "mucho»

\begin{tabular}{lcccc}
\hline & $\begin{array}{l}\text { Asistencia } \\
\text { a clase }\end{array}$ & $\begin{array}{l}\text { Trabajo } \\
\text { en grupo }\end{array}$ & $\begin{array}{l}\text { Conocimien- } \\
\text { tos prácticos y } \\
\text { metodológicos }\end{array}$ & $\begin{array}{l}\text { Teorías, } \\
\text { conceptos y } \\
\text { paradigmas }\end{array}$ \\
\hline Europa & 68 & 38 & 37 & 62 \\
España & 36 & 36 & 32 & 68 \\
Francia & 74 & 43 & 44 & 39 \\
Reino Unido & 84 & 34 & 51 & 53 \\
Alemania & 75 & 35 & 35 & 51 \\
México & 79 & 62 & 60 & 71 \\
\hline
\end{tabular}

Fuente: elaboración propia. 
Aunque con algunas diferencias, Francia, Alemania y Reino Unido otorgan mayor importancia a la "Asistencia a clase» que la media europea (se considera como Europa al conjunto total de países participantes en el proyecto REFLEX). Por contra, España es el país europeo en el que se le concede menos importancia. En todos los países, se enfatiza menos el «Trabajo en grupo» como método de enseñanza-aprendizaje que la «Asistencia a clase», con la excepción de España, donde ambos aspectos tienen una importancia similar.

En cuanto al balance entre "Conocimientos prácticos y metodológicos» y "Teorías, conceptos y paradigmas», se observan situaciones muy distintas. Mientras Francia y Reino Unido tienen un sistema educativo con estos dos aspectos equilibrados, en España se produce una diferencia muy grande a favor de la teoría y en detrimento de los aspectos prácticos. En México, se produce la misma situación, pero de modo mucho menos acusado.

Para relacionar simultáneamente los distintos métodos de enseñanza-aprendizaje con los distintos países, de modo que se evidencien con mayor detalle sus diferencias y sus similitudes, se ha realizado un análisis de correspondencias. Esta técnica estadística multivariante permite evaluar asociaciones entre unos y otros. El mapa que se presenta en la figura 2 muestra los resultados obtenidos.

Figura 2. Mapa de asociación entre los métodos de enseñanza-aprendizaje y los países

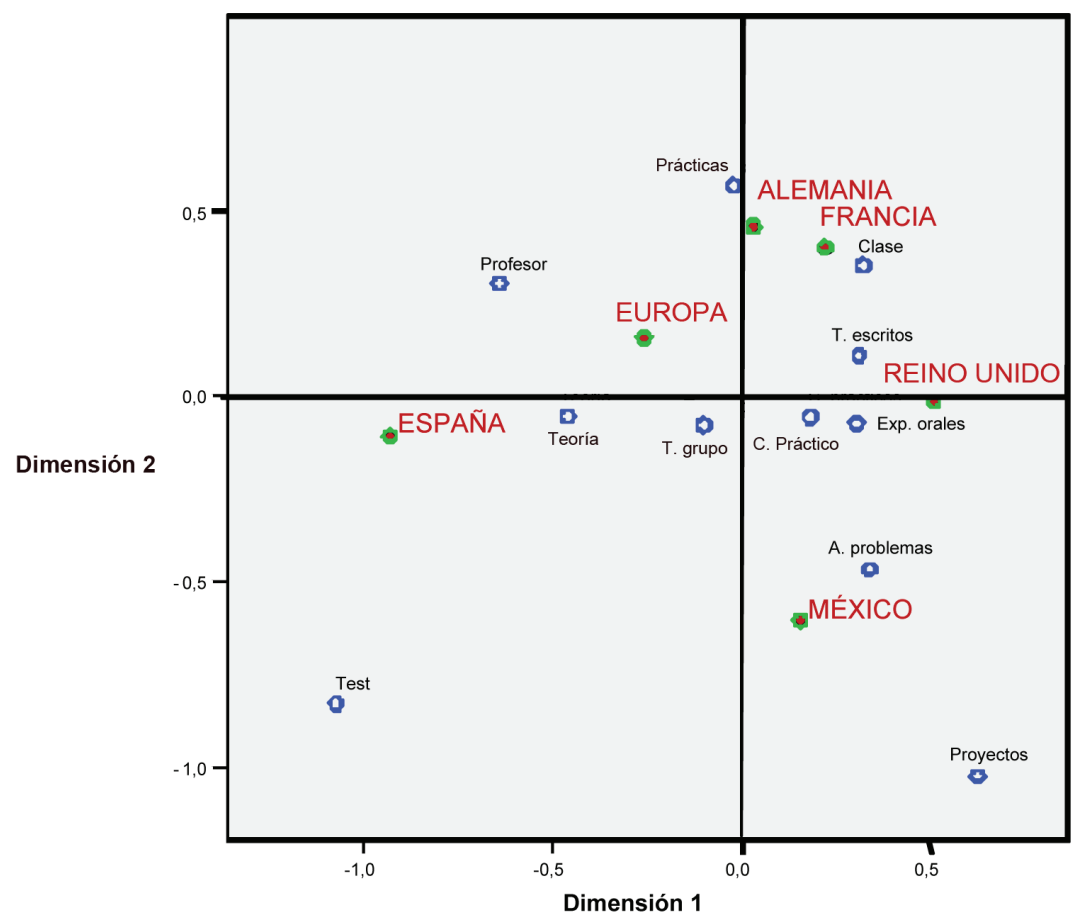

Fuente: elaboración propia. 
Lo que caracteriza a Reino Unido es la importancia que se le da a la «Asistencia a clase» y a los «Trabajos escritos» (esto lo comparte con Alemania y Francia), seguido de los «Conocimientos prácticos» (aquí con valores mayores que Alemania y Francia y, por supuesto, que España) y de la escasa importancia que se le da al «Profesor como principal fuente de información»: sólo el 33\% de los encuestados opinaron que éste fue un factor bastante o muy relevante en su aprendizaje, lo que supone el valor más bajo de Europa. En este país, a pesar de que más de la mitad de los entrevistados afirmaron que la "Teoría» era un aspecto muy relevante de sus estudios universitarios (52\%), dicho aspecto tiene una importancia por debajo de la media de Europa.

Alemania y Francia presentan cierta similitud en muchas cuestiones, destacan el énfasis en la "Asistencia a clase» (73\% de Francia frente a 75\% de Alemania), la importancia que se le concede al «Profesor como principal fuente de información" ( $51 \%$ de Francia frente a $44 \%$ de Alemania), el uso de «Exposiciones orales» (37\% y 43\%, respectivamente) o de «Trabajos escritos» ( $55 \%$ en ambos casos). Tampoco se encuentran grandes diferencias en el énfasis global de teoría y práctica.

En cuanto a España, lo que la caracteriza y la diferencia del resto es la enorme importancia que se da al «Profesor como principal fuente de información», el peso que se otorga al concepto de «Teorías, conceptos y paradigmas» frente a los «Conocimientos prácticos» y la poca importancia que se da a la «Asistencia a clase» (figura 3 ).

Figura 3. Los métodos de enseñanza-aprendizaje en España. Porcentaje de encuestados que ha respondido «bastante» $\mathrm{o}$ «mucho»

$\begin{array}{lllllllllll}0 & 10 & 20 & 30 & 40 & 50 & 60 & 70 & 80 & 90 & 100\end{array}$

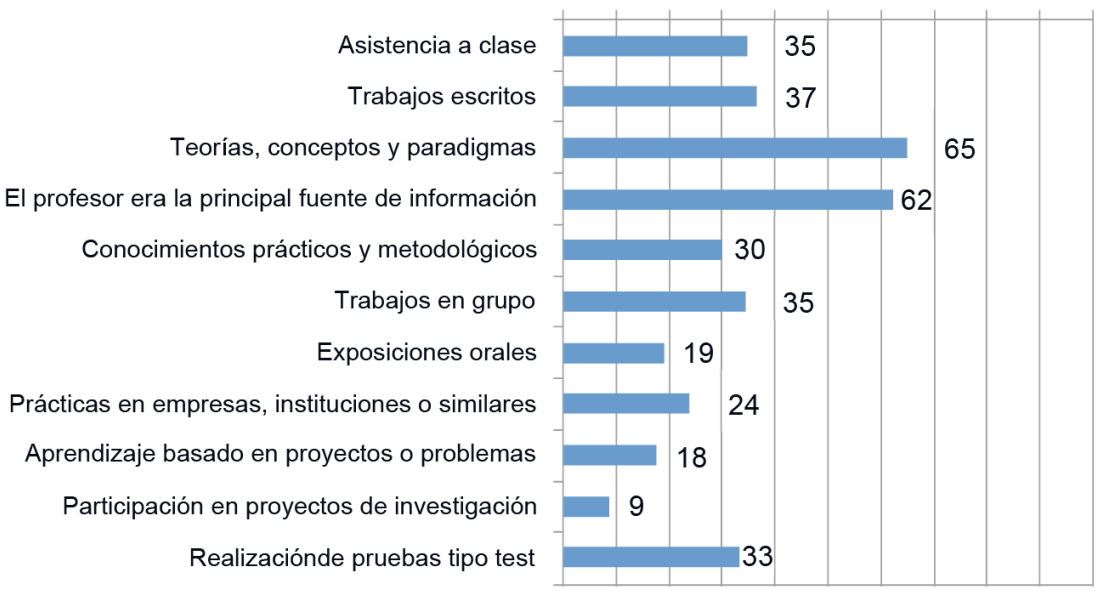

Fuente: elaboración propia. 
Finalmente, es necesario señalar que México es un país con diferencias claras frente a los países europeos, aunque comparte ciertas características con algunos de ellos. De forma general, su sistema educativo, en lo que a métodos de enseñanza-aprendizaje se refiere, se parece más a Reino Unido que a España. Un importante rasgo distintivo de México frente al resto de países es el uso del aprendizaje basado en proyectos o problemas y la participación en proyectos de investigación.

\section{Características de la carrera}

Se preguntó a los graduados que indicasen, en una escala graduada de 1 (nada) a 5 (mucho), en qué medida se podían aplicar una serie de características a sus estudios universitarios. En la tabla 2, se muestra por países la proporción de graduados según la principal característica de su carrera.

En cada país considerado, y en Europa en su conjunto, se aprecia que la proporción de titulados que afirman que sus estudios se caracterizaron en mayor medida por un enfoque generalista es muy similar a la de quienes consideran que había que estudiar mucho para aprobar. La única excepción es Francia, donde tan sólo el 38\% de los titulados respondieron que su carrera fue bastante o muy generalista, frente al $53 \%$, que consideraba que tenía que estudiar mucho para aprobar. Sin embargo, es en Francia donde parece que es necesario realizar un menor esfuerzo para superar los estudios universitarios. Por otra parte, los bajos porcentajes presentados en el prestigio académico en comparación con los mostrados en los otros dos aspectos examinados podrían sugerir que ni el enfoque generalista ni el esfuerzo para aprobar son factores que contribuyan al prestigio de las titulaciones universitarias. En esta ocasión, la excepción es México, donde el prestigio de la carrera es el aspecto más destacado por los graduados universitarios.

\section{Comportamiento de los estudiantes durante la carrera}

A continuación, se examina la respuesta de los titulados a varias cuestiones relacionadas con su actividad y estrategia mientras cursaban su carrera uni-

Tabla 2. Características de la carrera. Porcentaje de graduados que respondieron «bastante» o «mucho»

\begin{tabular}{lccc}
\hline & Enfoque generalista & $\begin{array}{l}\text { Carrera con prestigio } \\
\text { académico }\end{array}$ & $\begin{array}{l}\text { Había que estudiar } \\
\text { mucho para aprobar }\end{array}$ \\
\hline Europa & 64 & 47 & 63 \\
España & 66 & 53 & 67 \\
Francia & 38 & 23 & 53 \\
Reino Unido & 58 & 49 & 66 \\
Alemania & 69 & 39 & 61 \\
México & 73 & 81 & 71 \\
\hline
\end{tabular}

Fuente: elaboración propia. 
Figura 4. Relación entre horas dedicadas al estudio y porcentaje de encuestados que respondió «bastante» $\mathrm{o}$ «mucho» al esfuerzo percibido para aprobar

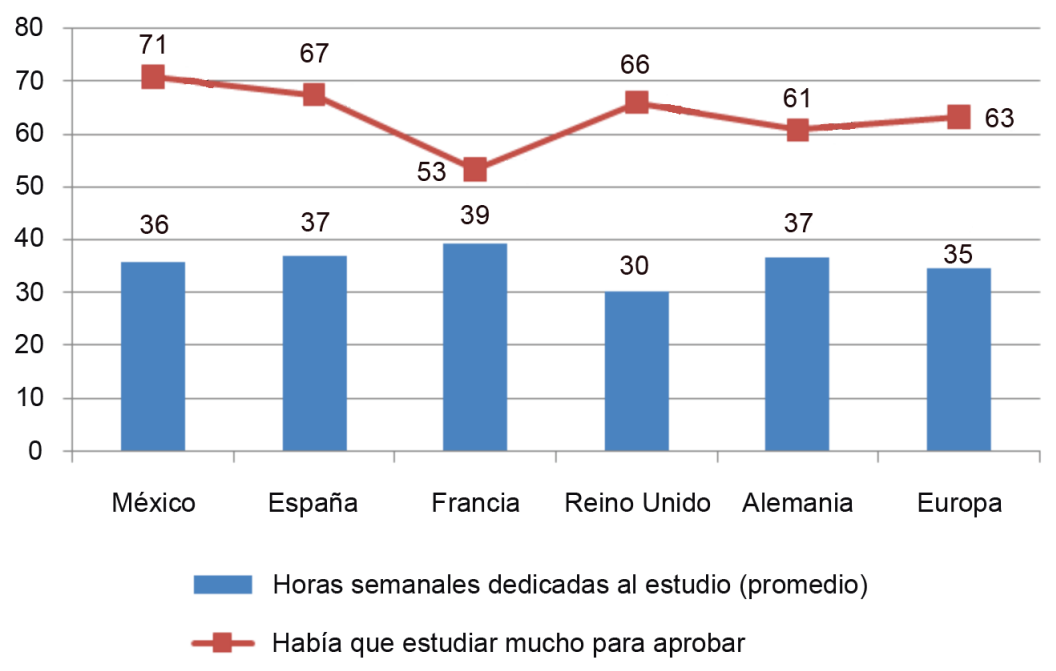

Fuente: elaboración propia.

versitaria. Entre ellas, el promedio de horas semanales dedicadas al estudio, que, en la figura 4, se relaciona con el esfuerzo percibido por los graduados en cada país. En las horas semanales dedicadas a actividades relacionadas con sus estudios, se incluyen asistencia a clase, trabajos en grupo, estudio personal, trabajo en biblioteca, etc.

Se observan notables diferencias en las horas semanales según países, pero sobre todo entre la relación en cantidad de horas y esfuerzo percibido por el estudiante. Resulta paradójico que un país como Francia presente el valor promedio de horas semanales dedicadas al estudio más alto junto con el valor más bajo en cuanto a los graduados que han manifestado que recuerdan que había que estudiar bastante o mucho para aprobar. La situación contraria se observa en Reino Unido, donde el menor número de horas de estudio se combina con un alto valor de esfuerzo percibido. A pesar de la extrañeza que causan estos resultados, deben leerse en el contexto de modelos universitarios muy distintos, como el napoleónico o el anglosajón.

La tabla 3 muestra el número medio de horas semanales de estudio por países y áreas. Los titulados que destacan por haber estudiado más horas por término medio, en todos los países, son los que cursaron una carrera del área de salud, seguidos por los graduados en ciencias o técnicas. No obstante, el número medio de horas que en Alemania destinaron los estudiantes de las carreras técnicas fue similar al empleado por los graduados en educación, derecho o economía y empresa. Por el contrario, son los egresados en el área de ciencias sociales quienes destacan por ser unos de los que menos horas destinaron al estudio. 
Tabla 3. Número de horas dedicadas al estudio

\begin{tabular}{lccccc}
\hline & México & España & Francia & Reino Unido & Alemania \\
\hline Educación & 32 & 35 & 38 & 29 & 36 \\
Humanidades & 40 & 36 & 34 & 30 & 33 \\
C. sociales & 33 & 34 & 34 & 27 & 31 \\
Econ. y emp. & 35 & 35 & 39 & 28 & 35 \\
Derecho & 30 & 34 & 38 & 27 & 36 \\
Técnicas & 40 & 42 & 44 & 32 & 38 \\
Salud & 43 & 42 & 45 & 34 & 46 \\
Ciencias & 43 & 38 & 44 & 32 & 36 \\
\hline
\end{tabular}

Fuente: elaboración propia.

Otro aspecto interesante en el comportamiento de los graduados es el porcentaje que ha cursado estudios adicionales a los finalizados en el año académico 1999-2000, ya que podría reflejar tanto la existencia de carencias del sistema educativo, cuando el graduado necesita formación adicional para poder acceder a determinados puestos de trabajo, como la dificultad en la inserción laboral de los jóvenes universitarios. Mientras que en Europa el porcentaje de graduados que ha realizado estudios adicionales muestra un promedio del $44 \%$, en España es del 56\%. Reino Unido (42\%) y Alemania (44\%) presentan valores cercanos a la media, mientras que Francia la supera (51\%). México, una vez más, presenta una situación distinta, con un porcentaje menor $(38 \%)$, valor que refleja una situación diferente a la europea en cuanto al acceso al mercado laboral de los graduados universitarios.

\section{Evaluación de los estudios realizados}

Esta sección analiza la visión retrospectiva de los graduados sobre la utilidad de su carrera y la evaluación que realizan de su elección de estudios. La utilidad de la carrera como una buena base para realizar diversas actividades vitales y profesionales es valorada por el entrevistado a través de una escala graduada de 1 (nada) a 5 (mucho). Por otra parte, para evaluar la elección de estudios, se preguntaba a los graduados cuál sería su decisión si pudieran volver atrás y fueran libres para elegir de nuevo.

La figura 5 muestra la proporción de quienes contestaron que su carrera ha sido una base bastante o muy buena (valores 4 y 5 de la escala) en el desarrollo de los distintos aspectos examinados. Dichas proporciones se presentan para el conjunto de países europeos participantes en el proyecto REFLEX, ya que, en general, no se han encontrado grandes discrepancias entre ellos. Además, tanto en Europa como en México, el orden en que los graduados han evaluado los distintos aspectos es casi idéntico. El diferente grado en el porcentaje de respuesta podría deberse a diferencias sociales y/o culturales.

En Europa, la mayoría de los graduados entrevistados señalaba que sus respectivas carreras constituían una buena base para «Mejorar tu desarrollo 
Figura 5. Evaluación de la carrera como base. Proporción total de graduados que respondió «bastante» o «mucho». Promedio europeo

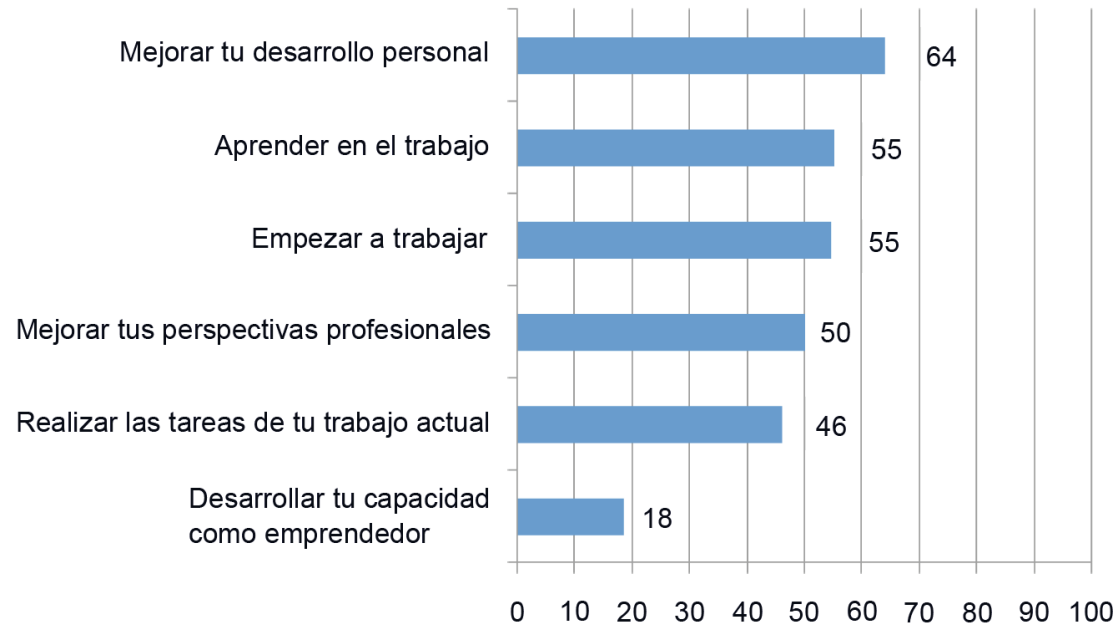

Fuente: elaboración propia.

personal» $(64 \%)$, para "Aprender en el trabajo» (55\%) y para «Empezar a trabajar» (55\%). La mitad de los graduados consideraba su carrera como base para mejorar sus perspectivas profesionales, mientras que un $46 \%$ opinaba que sus estudios eran una buena base para «Realizar las tareas de tu trabajo actual». En el extremo inferior, sólo el 18\% de los graduados opinaba que su carrera había servido para «Desarrollar tu capacidad como emprendedor».

Finalmente, como indicador resumen de la valoración global de los graduados acerca de su paso por la universidad, se preguntaba sobre la elección de sus estudios y de su universidad. La figura 6 muestra los resultados en cada uno de los países considerados y refleja algunas diferencias entre ellos, aunque, en conjunto, los graduados se mostraban satisfechos con la elección de carrera y con la universidad que escogieron. Más de la mitad de los graduados encuestados en todos los países declaraba que volvería a cursar la misma carrera en la misma universidad si pudiese volver atrás y fuese libre para elegir. En todos los países, más del 70\% escogería la misma universidad. En el caso de México, se llegó al 86\%. En el extremo contrario, encontramos bajos porcentajes de encuestados que respondieron que no estudiarían una carrera universitaria. España, con un 9\%, es el país en el que este porcentaje es notablemente mayor. Una vez más, la opinión de los graduados españoles refleja notables diferencias con la media europea y con los países representativos de los modelos educativos clásicos. En conjunto, la respuesta a esta pregunta puede ser interpretada como una medida de la satisfacción global de los graduados con los estudios universitarios que cursaron en su día desde la perspectiva que les conceden los cinco años transcurridos hasta el momen- 
Figura 6. Evaluación retrospectiva de la elección de carrera. Proporción total de graduados que elegiría cada opción

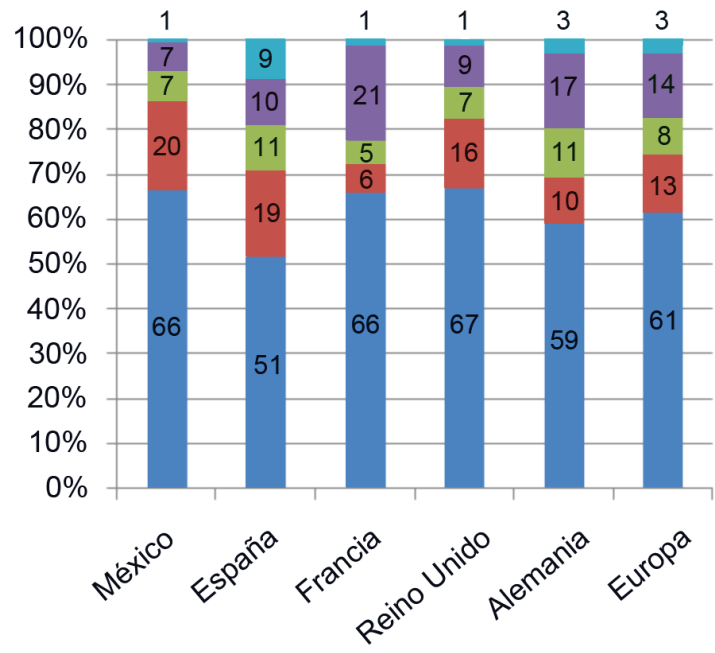

- No estudiaría una carrera universitaria.

- Una carrera diferente en otra universidad.

- La misma carrera en otra universidad.

Una carrera diferente en la misma universidad.

- La misma carrera y la misma universidad.

Fuente: elaboración propia.

to de la entrevista. En este sentido, los titulados españoles son, en términos generales, los menos satisfechos.

\section{Las competencias en España}

Los graduados españoles indican en sus respuestas que los estudios universitarios cursados han contribuido de manera globalmente relevante, aunque de forma muy desigual, a la adquisición o desarrollo de las diecinueve competencias consideradas en la encuesta. Genéricamente, y en términos de valores medios de una escala de 1 a 7 , tan solo cinco competencias presentan valores medios de la contribución de la universidad más elevados que el valor central de la escala: "Capacidad para adquirir con rapidez nuevos conocimientos» $(4,39)$, "Capacidad para trabajar en equipo" (4,33), "Capacidad para redactar informes o documentos» $(4,10)$, «Pensamiento analítico» $(4,09)$ y «Dominio del área o disciplina» $(4,07)$. Estas serían, por tanto, las competencias que los graduados reconocen haber desarrollado gracias a sus estudios universitarios en mayor medida. Por el contrario, la menor contribución absoluta de la universidad corresponde a la competencia «Capacidad para escribir y hablar en idiomas extranjeros» $(2,22)$, seguida a cierta distancia por la "Capacidad para negociar de forma eficaz» $(2,85)$ y la "Capacidad para hacer valer tu autoridad» $(2,98)$. La opinión de los graduados revela que la contribución de los estudios universitarios ha sido muy limitada en cuanto a la adquisición de estas competencias. Para el resto de competencias consideradas, la contribución media de los estudios universitarios oscila entre los valores 3 y 4 de la escala. 
Figura 7. Relación entre la contribución de la universidad para formar competencias y el nivel propio de los graduados en el momento de realizar la encuesta

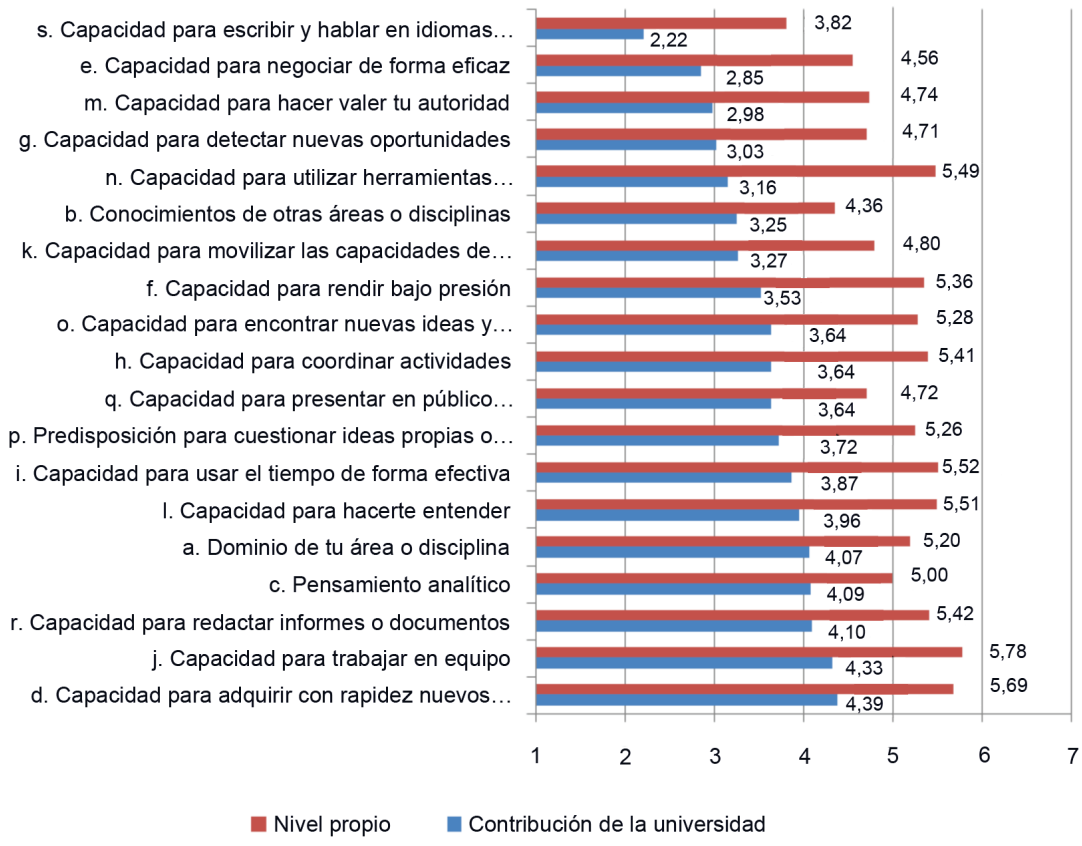

Fuente: elaboración propia.

La contribución de la universidad a la provisión de las diversas competencias puede relativizarse considerándola de manera conjunta con el nivel competencial propio de los graduados en el momento de realizar la entrevista. La discrepancia entre la contribución de la universidad y el nivel de competencia propio de los graduados indica en qué medida las diversas competencias han sido desarrolladas por otros medios, tales como la experiencia profesional o diversas formas de educación no universitaria. Globalmente, se observa que el nivel competencial propio de los graduados españoles es más elevado en aquellas competencias con alta contribución de la universidad que en las que presentan una contribución baja, aunque se aprecian diferencias substanciales entre unas competencias y otras. Así, entre las primeras destaca la competencia «Pensamiento analítico», para la que el nivel propio se encuentra sólo un punto por encima de la contribución de los estudios, hecho que sugiere que, en este ámbito, la contribución de la universidad es crucial y que los mecanismos alternativos de desarrollo de competencia desempeñan un papel mucho más limitado. Algo semejante sucede, aunque a menor escala, con las competencias "Dominio de tu área o disciplina» y "Capacidad para presentar en público productos, ideas e informes» $y$, entre las competencias que presentan una contribución absoluta más reducida de la universidad, con los «Conocimientos de 
otras áreas o disciplinas». Por el contrario, las competencias que los graduados han desarrollado en mayor medida a través de mecanismos no vinculados a sus estudios universitarios se encuentran, por lo general entre aquellas en que su nivel propio es comparativamente bajo. Destacan especialmente la «Capacidad para escribir y leer en idiomas extranjeros» y la "Capacidad para utilizar herramientas informáticas».

\section{Conclusiones}

El análisis descriptivo desarrollado en este estudio tiene como objetivo principal proporcionar información relevante para los gestores educativos universitarios, ya que la inserción en el Espacio Europeo de Educación Superior requiere que estos conozcan con gran precisión la situación en la que se encuentra la universidad actual, a fin de elaborar los nuevos planes de estudio. Para ello, utilizando datos procedentes del proyecto internacional REFLEX (en él participan trece países europeos: Italia, España, Francia, Austria, Alemania, Países Bajos, Reino Unido, Finlandia, Noruega, República Checa, Suiza, Bélgica y Estonia), se desarrolla un análisis comparativo sobre la metodología de aprendizaje, las características de la carrera, la dedicación de los titulados en las misma y la contribución de los estudios cursados en el desarrollo vital y profesional del egresado en España y en tres países que representan tres modelos tradicionales de universidad: Alemania (humboldtiano), Francia (napoleónico) y Reino Unido (anglosajón). Por otra parte, también se considera en el análisis a México, como país no europeo cuyo modelo de universidad está influenciado por diversas tendencias. Adicionalmente, para el caso español, se analizan las competencias profesionales proporcionadas por los estudios universitarios, así como las poseídas por los titulados cinco años después de la graduación.

A continuación, se remarcan algunos de los rasgos más relevantes sobre la visión que los graduados tienen de sus estudios universitarios:

- Se han encontrado grandes diferencias en los métodos de enseñanza-aprendizaje ente los distintos países, reflejo de modelos universitarios distintos. Concretamente, en España, destaca la relevancia del profesor y la enseñanza teórica como fuentes principales de información, mientras que el Reino Unido, Alemania y Francia se caracterizan por la importancia otorgada a la asistencia a clase y a los conocimientos prácticos. Por su parte, México destaca por utilizar una metodología de aprendizaje basada en proyectos o problemas y la participación en proyectos de investigación.

- Excepto en Francia, la proporción de graduados que consideran que su carrera universitaria se caracterizó por un elevado enfoque generalista es elevada y muy similar a la de quienes afirman que debían estudiar mucho para aprobar. Sin embargo, excepto en México, la proporción de titulados que afirman que su carrera gozaba de prestigio académico es muy inferior a la de los otros dos aspectos mencionados, sugiriendo que ni el enfoque 
generalista ni el esfuerzo para aprobar son factores que contribuyen al prestigio universitario. Es México el país donde el porcentaje de titulados en carreras con prestigio es mayor.

- Junto con Francia y Alemania, España es uno de los países donde los titulados dedicaron más horas de estudio. Frente a las treinta y siete horas del caso español, en países con estudios superiores altamente valorados a nivel internacional, como Reino Unido, la dedicación semanal de un estudiante universitario está en torno a las treinta horas por término medio.

- Los titulados que más tiempo destinan a estudiar son los que cursaron una carrera del área de salud, ciencias o técnica, contrariamente a lo que sucede entre los titulados en ciencias sociales.

- La proporción de titulados universitarios que afirman haber adquirido formación adicional a la universitaria es superior en Francia (51\%) y, sobre todo, en España (56\%) a la media de los países europeos participantes en el proyecto REFLEX (44\%). Dichos porcentajes alcanzan el 42\% en Reino Unido, el $44 \%$ en Alemania y el 38\% en México.

- En general, los titulados de los distintos países europeos participantes en el proyecto REFLEX afirman que sus estudios universitarios fue una base bastante o muy buena para "Mejorar el desarrollo personal», "Aprender en el trabajo» y «Empezar a trabajar». Sin embargo, no tuvo gran relevancia para "Desarrollar la capacidad de emprendedor".

- Pese a las diferencias encontradas entre los distintos sistemas universitarios examinados, los titulados de los distintos países afirman en su gran mayoría que volverían a estudiar su misma carrera en su misma universidad. El dato más sorprendente es que el $9 \%$ de los titulados españoles exponen que, si pudieran elegir de nuevo su trayectoria académica, no volverían a estudiar una carrera universitaria. Dicho porcentaje es del $1 \%$ en México, Francia y Reino Unido y del 3\% en Alemania.

- Sin realizar una valoración comparativa de la calidad de los contenidos de los estudios cursados por los titulados de los países analizados, en términos generales, los graduados españoles son los menos satisfechos con los estudios universitarios cursados.

- En opinión de los graduados, los estudios universitarios contribuyeron de manera crucial a ayudarles a adquirir competencias cognitivas y metacognitivas, mientras que su contribución a la adquisición de competencias instrumentales fue muy limitada.

\section{Referencias bibliográficas}

Belfield, C. R.; Bullock, A. D.; Fielding, A. 1999. "Graduates' view on the contribution of their higher education to their general development: a retrospective evaluation for the United Kingdom». Research in Higher Education, 40 (4), 409-38.

EUROPEAN COMMISSION. 2005. «Strategy for an innovative and inclusive European Information Society». Information Society and Media. General Fact and Sheet.

HARTOG, J. 2001. "On human capital and individual capabilities». Review of Income and Wealth, 47 (4), 515-40. 
Kellerman, P. 2007. «Acquired and required competencies of graduates». En: U. Teichler (ed.). Careers of university graduates: views and experiences in comparative perspectives. Dordrecht: Springer.

KnABB, S. D.; STODDARD, C. 2005. "The quality of education, educational institutions, and cross-country differences in human capital accumulation». Growth and Change, 36, 354-73.

MenG, C.; HeIKE, H. 2005. «Student time allocation, the learning environment, and the acquisition of competencies». ROA Research Memorandum ROA-RM2005/1E. Maastricht University.

MorA, J. G.; CEGES-LMPF. 2008. «El “éxito laboral” de los jóvenes graduados universitarios europeos». Revista de Educación, no extraordinario: 41-58.

REFLEX. 2009. http:// www.reflexproject.org

SCHOMbuRG, H. 2007. «The professional success of higher education graduates». European Journal of Education, 42 (1), 35-57.

VilA, L. E.; GarCÍA-Aracil, A.; MorA, J. G. 2007. «The distribution of job satisfaction among young European graduates: Does the choice of study fiel matter?». The Journal of Higher Educatio, 78, 97-118. 